\title{
LA RELACIÓN ENTRE EL USO DE LAS NTIC EN LA COMPETITIVIDAD EN LAS MICRO Y PEQUEÑAS EMPRESAS COMERCIALES DE GUAYAQUIL
}

THE RELATION BETWEEN THE USE OF NTIC IN THE
COMPETITIVENESS OF MICRO AND SMALL ENTERPRISES OF
GUAYAQUIL

María Eugenia Jiménez Gercado

Docente, Universidad de Guayaquil, (Ecuador).

E-mail: jimenezcme@ug.edu.ec ORCID: https://orcid.org/0000-0003-1404-6731

Marjorie Marcela Acosta Véliz

Docente, Universidad de Guayaquil, (Ecuador).

E-mail: marjorie.acostav@ug.edu.ec ORCID: https://orcid.org/0000-0002-9462-1881

Diego Iván Muñoz Naranjo

Docente, Universidad Agraria del Ecuador, (Ecuador).

E-mail: dmunoz@uagraria.edu.ec ORCID: https://orcid.org/0000-0003-2203-0588

Evelyn García Moreira

Docente, Universidad de Guayaquil, (Ecuador).

E-mail: evelyn.garciam@ug.edu.ec ORCID: https://orcid.org/0000-0002-2181-3364

Recepción: 28/04/2020 Aceptación: 15/06/2020 Publicación: 29/06/2020

Gitación sugerida:

Jiménez, M. E., Acosta, M. M., Muñoz, D. I., y García, E. (2020). La relación entre el uso de las NTIC en la competitividad en las micro y pequeñas empresas comerciales de Guayaquil. 3C TIC. Cuadernos de desarrollo aplicados a las TIC, 9(2), 119-137. https://doi.org/10.17993/3ctic.2020.92.119-137 


\section{RESUMEN}

El presente proyecto tiene por objeto mostrar de qué modo el uso de las NTIC (Nuevas Tecnologías de la Información y Comunicación) influye o se relaciona con la competitividad de las MiPYME (Micro, Pequeña y Mediana Empresa) del sector comercial de Guayaquil, la ciudad más próspera del Ecuador. La metodología utilizada contempla lo descriptivo, exploratorio y documental además de datos que son obtenidos de fuentes bibliográficas y de entes avalados por el Estado donde reposa la información concerniente al entorno económico del país. ¿Es verdaderamente útil para la supervivencia de las empresas la asimilación de las NTICs? ¿Son aplicables dentro del contexto financiero ecuatoriano? Desafortunadamente, a pesar de que la tecnología tiene un lugar importante dentro de los diferentes roles empresariales y que en otros países ya se han vuelto parte de su cultura y cotidianeidad, en las comerciales guayaquileñas aún presentan dificultades para su adaptación; estas barreras incluyen desde la falta de capital para realizar inversiones de infraestructura que permita la digitalización de la información y demás herramientas, hasta la falta de conocimientos avanzados que ayuden a elegir oportunamente lo más adecuado para el estilo de negocio que se lleva a cabo. Finalmente, los resultados reflejan que la aplicación de las NTICs es necesaria para mejorar la competitividad de las MiPYMEs, caso contrario, las empresas perecerán en el mercado que percibe el uso de tecnología como sinónimo de calidad y prestigio. Por ende, se concluye que existe una alta relación entre las NTICs y las MiPYMEs comerciales de Guayaquil.

\section{PALABRAS CLAVE}

NTIC, Microempresa, Pequeña empresa, Mediana empresa, Competitividad. 


\section{ABSTRACT}

The purpose of this project is to show how the use of the NTIC (New Information and Communication Technologies) influences or is related to the competitiveness of the MSMEs (Micro, Small and Medium-sized Enterprises) of the commercial sector of Guayaquil, the most prosperous city from Ecuador. The methodology used includes the descriptive, exploratory and documentary, in addition, also data that is obtained from bibliographic sources and from entities endorsed by the State where the information regarding of the economic environment of the country lies. $i$ Is assimilation of NTICs really useful for the survival of companies? 'Are they applicable within the Ecuadorian financial context? Unfortunately, despite the fact that technology has an important place within the different business roles and that in other countries they have already become part of their culture and daily life, in the commercial MSMEs in Guayaquil they still present difficulties in their adaptation; These barriers range from a lack of capital to make infrastructure investments that allow the digitization of information and other tools, to a lack of advanced knowledge that helps to choose in a timely manner what is most appropriate for the style of business being carried out. Finally, the results reflect that the application of the NTICs is necessary to improve the competitiveness of MSMEs, otherwise, companies will perish in the market that perceives the use of technology as a synonym of quality and prestige. Therefore, it is concluded that there is a high relationship between the NTICs and the commercial MSMEs of Guayaquil.

\section{KEYWORDS}

NTIC, Micro enterprise, Small enterprise, Medium enterprise, Competitiveness. 


\section{INTRODUCTION}

En el presente trabajo se analizará la relación que tiene el uso de las NTICs con la competitividad de las MiPYME (Micro, Pequeñas y Medianas Empresas) comerciales de Guayaquil. Es necesario, por tanto, definir brevemente las siglas que se utiliza: TICs se refiere a Tecnologías de la Información y Comunicación y NTIGs indica que son Nuevas Tecnologías de la Información y Comunicación. En ambos casos, dentro del marco teórico se detallará su valioso aporte a la competitividad empresarial.

Es innegable, que la tecnología ha tenido una alta repercusión en los últimos años en diferentes ámbitos, no solo en lo social y ambiental, sino también en los sectores económicos de los países, más aún en el área empresarial, base del desarrollo financiero. Por un lado, las empresas invierten en adelantos tecnológicos para liderar los mercados, por otro lado, las empresas que no invierten en tecnología, se quedan retraídas en el "pasado" perdiendo competitividad por ende clientes y posteriormente se aíslan de un mundo globalizado.

La información constituye una herramienta esencial para formar criterios que posteriormente recaerán en toma de decisiones, es por tanto, que actualmente se considera un beneficio el buen manejo de la misma. Consecuentemente, las empresas buscan técnicas que faciliten la administración de este recurso con la más absoluta cotidianeidad, y realizan inversiones económicas que, organizaciones más experimentadas, ya han logrado visionar, palpar y diseñar caminos cuyas ventajas crean competitividad y liderazgo.

Entre tanto, la comunicación es esa fuerza poderosa de mantener al tanto de los movimientos, decisiones y muchos elementos importantes, a todas las áreas estratégicas de una empresa. Una verdadera comunicación es clave de éxito de empresas líderes, productos apetecidos por clientes, ventas crecientes y buenos gerentes. Superar los problemas caracteriza al ser humano, así mismo la comunicación; no es sencillo, nunca lo ha sido, pero sí que ha ayudado la tecnología. Sin embargo, el flujo de comunicación puede volverse tedioso y abrumador. 
En Ecuador, en el 2017, existían mayormente 90.78\% de micro empresas, lo cual es beneficioso para la economía local, proporcionando liquidez inmediata a diferentes negocios, que en algunos casos son familiares. Constituyen merecidamente más del 60\% de la fuerza laboral del país, esto destaca el espíritu emprendedor de este país, que por diversos motivos busca crear plazas de trabajo que alberguen gente joven con ideas innovadoras y que a su vez impulsen más negocios acogiéndose a las tendencias del mundo globalizado.

Las pequeñas empresas alcanzan el 7.22\%, que sumándose al porcentaje de las micro empresas, logran crear un valioso aporte al PIB (Producto Interno Bruto) nacional. Dentro del sector comercial están 319.503 empresas, las mismas que se caracterizan por obedecer las necesidades más próximas del mercado, así como también de las tendencias a nivel mundial. Esto las hace más flexibles al momento de aplicar nuevas tecnologías, se adaptan y se vuelven competitivas aunque sea menos fácil acceder a créditos bancarios dadas las garantías que exige el sector financiero.

Las micro y pequeñas empresas generalmente empiezan con recursos propios por lo cual las fuentes de financiamiento externa son escasas o dificiles de lograr, sin embargo, por medio de las TICs, es posible la optimización de los recursos como lo son el tiempo y dinero, mejorando así el rendimiento de la empresa. Es necesario también indicar que las herramientas de las TICs agilizan los procesos organizacionales, automatizando diferentes gestiones que van desde lo administrativo hasta lo financiero facilitando así la maximización de la competitividad.

Las TICs como herramienta de apoyo a las empresas, brinda la ventaja de la diferenciación a través de mecanismos visuales, orales o escritos con ayuda de técnicas de marketing. La información inclusive será tratada eficientemente y comunicada a usuarios internos y externos para su respectivo registro y análisis, consecuentemente, la generación e implementación de estrategias vendrán de manera positiva para posicionar a la marca o producto de manera constante dentro de mercados competitivos y cambiantes 
De acuerdo al estudio realizado por Guzmán, Guzmán, y Fuentes (2016), en el cual se muestra el grado de digitalización que poseen las PYMES (Pequeñas y Medianas Empresas) guayaquileñas en cuanto al área económica y de comunicación dentro de su entorno comercial, se detecta interacciones primordialmente con bancos, clientes, proveedores y empleados. Como se aprecia en la Figura 1, la aplicación de las TIC por parte de las pyme guayaquileñas, se encuentran en una etapa media de adopción.

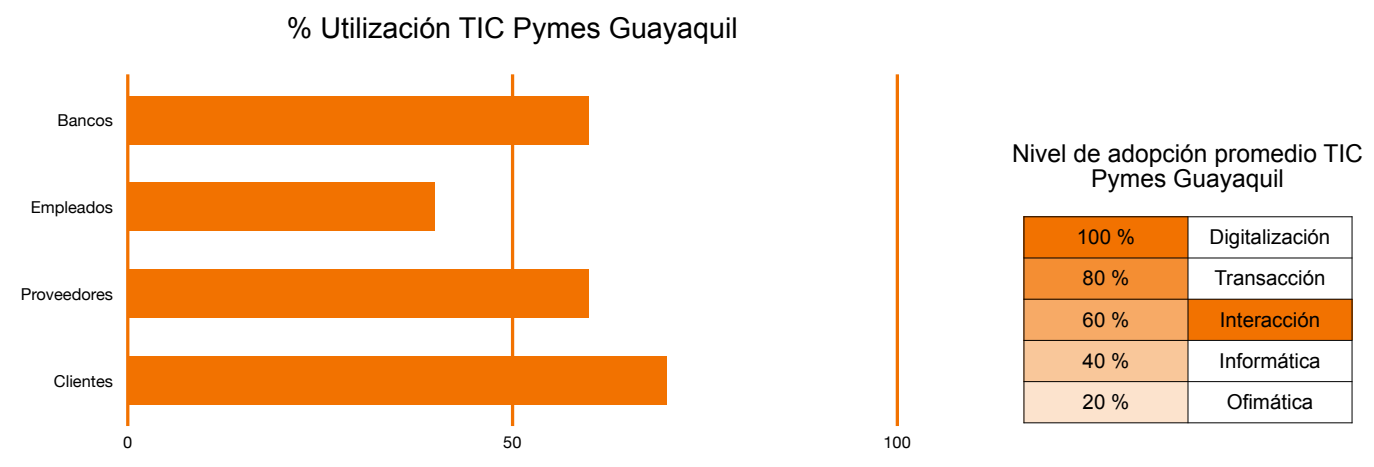

Figura 1. Adopción de las TIC en las PYMES (Pequeña y Mediana Empresa) guayaquileñas por segmento y total. Fuente: (Guzmán, Guzmán, y Fuentes, 2016).

\section{METODOLOGÍA}

$\mathrm{Al}$ analizar el lugar de las NTICs y sus particularidades en la empresa ecuatoriana, se toma información verídica, factible y adecuada que se torna relevante al aplicarla como metodología de este proyecto. Los instrumentos utilizados en la presente investigación así como sus métodos contemplan un enfoque mixto, entre ellos se encuentra el estudio descriptivo que según Hernández y Fernández (2003); "sirven para especificar propiedades, características y rasgos importantes del fenómeno a analizar".

Adicionalmente, se aplica el estudio exploratorio, dado que como lo indica Hernández y Fernández (2003); "tienen como objetivo esencial examinar un tema poco estudiado", es decir, los temas de NTICs son abordados día a día en diferentes ámbitos de escenarios futuristas y de vanguardia, de igual forma la competitividad empresarial ha tenido un sinnúmero de estudios. Sin embargo, este trabajo busca la 
relación de las nuevas tecnologías de la información y comunicación dentro de la competitividad de las micro y pequeñas empresas comerciales de Guayaquil.

También se aplica el estudio documental, que según Lara (2011) “consiste en un análisis de la información escrita sobre un determinado tema, con el propósito de establecer relaciones, diferencias, etapas, posturas o estado actual del conocimiento respecto al tema". Por consiguiente, es oportuno este método ya que se estudia la relación de las NTICs con la competitividad de las micro y pequeña empresas comerciales de Guayaquil, se utiliza información ya publicada y sustentada cuyos insumos contribuyen al desarrollo de la investigación.

Por un lado, se requiere el método inductivo descrito por Muñoz (2011) "cómo razonamiento que analiza una porción de un todo" en otras palabras, se estudia lo específico o particular para llegar a un conocimiento general. Por otro lado, con el método deductivo que también lo describe Muñoz (2011); "es el razonamiento que parte de un marco general de referencia hacia algo en particular", se utiliza para ir de conocimientos universales para llegar a lo individual.

Para finalizar, se utilizan los métodos analítico y sintético, descritos por Lopera y Ramírez (2010). El analítico estudia individualmente las partes como los hechos del objetivo del proyecto y el sintético revisa el proceso de la relación de estos hechos Consecuentemente, los métodos antes citados han aportado a la investigación dado que permiten analizar por separado sus componentes para luego juntar las partes y así obtener una idea general del fenómeno. Ventajosamente, ayudan a visualizar el entorno del problema para luego identificar sus partes y características, de este modo se podrá mostrar cómo se relacionan entre sí en diferentes ámbitos. 


\section{RESULTADOS}

\section{Las Micro, Pequeñas y Medianas empresas}

Las Micro, Pequeñas y Medianas empresas según la Comunidad Andina de Naciones (CAN) se encuentran divididas en estratos económicos con base en la cantidad de trabajadores que laboran y sus ingresos brutos (Cámara de Comercio de Quito, 2017). Los cuales se pueden observar en la Tabla 1.

Tabla 1. Clasificación de las Micro, Pequeñas y Medianas empresas según la CAN.

\begin{tabular}{|c|c|c|}
\hline Variables & Personal ocupado & Valor bruto de ventas anuales \\
\hline Estrato 1 & $1-9$ & $\leq$ USD 100.000 \\
\hline Estrato 2 & $10-49$ & USD 100.001-1'000.000 \\
\hline Estrato 3 & $50-99$ & USD 1'000.001-2'000.000 \\
\hline Estrato 4 & $100-199$ & USD 2'000.001-5'000.000 \\
\hline
\end{tabular}

Fuente: (Cámara de Comercio de Quito, 2017, pág. 1).

Sin embargo, la Superintendencia de Compañías, Valores y Seguros con base a la disposición de la CAN determinó la clasificación de las MiPYME Micro Pequeña y Mediana Empresa, como se muestra en la Tabla 2.

Tabla 2. Clasificación de las empresas según la Superintendencia de Compañías, Valores y Seguros.

\begin{tabular}{|c|c|c|c|}
\hline $\begin{array}{c}\text { Variables } \\
\text { Microempresa }\end{array}$ & Personal ocupado & Valor bruto ventas anuales & Monto de activos \\
\hline $\begin{array}{c}\text { Pequeña } \\
\text { empresa }\end{array}$ & $1-9$ & S USD 100.000 & Hasta USD 100.000 \\
\hline $\begin{array}{c}\text { Mediana } \\
\text { empresa }\end{array}$ & $10-49$ & USD 100.001-1'000.000 & De USD 100.001-750.000 \\
\hline Grande empresa & $20-199$ & USD 1'000.001-5'000.000 & $\begin{array}{c}\text { De USD 750.001- } \\
\text { 3'999.999 }\end{array}$ \\
\hline
\end{tabular}

Fuente: (Cámara de Comercio de Quito, 2017, pág. 2).

Según datos del Instituto Nacional de Estadística y Censos (INEC) para el 2017 las empresas registradas en el Ecuador fueron de 884.236 de las cuales el 90.78\% (802.696) son micro empresas, el 7.22\% 
(63.814) son pequeñas empresas, el 1.55\% (13.693) son medianas empresas y solo el $0.46 \%$ (4.033) son grandes empresas (Instituto Nacional de Estadística y Censos, 2018). Además, el 40.84\% (361.149) de las empresas se dedican a la prestación de algún tipo de servicio, el 36.13\% (319.503) se dedican al comercio, siendo estos sectores los más productivos.

Para Delgado y Chávez (2018), en Ecuador, las PYME (Pequeña y Mediana Empresa) generan el 60\% de las plazas laborales en el país, además son las encargadas de dinamizar la economía local. No solo su aportación al PIB es importante, también es su flexibilidad y fácil adaptación a los cambios en el mercado y el mundo, como la aplicación de nuevas tecnologías y tendencias sociales. Sus principales fuentes de financiamiento son los créditos bancarios y recursos propios, pero el mayor problema que enfrentan al tratar de crecer es la falta de cumplimiento en los requisitos para el apalancamiento financiero.

\subsection{LAS TIC}

Las Tecnologías de la Información y la Comunicación son todos aquellos desarrollos tecnológicos que permiten facilitar, agilizar y automatizar diversas funciones a fin de optimizar y maximizar el rendimiento de los recursos. Inicialmente la implementación de las TIC se destinó al mejoramiento de la enseñanza en escuelas, colegios y universidades. Sin embargo, su aplicación en las empresas se ha hecho cada vez más necesario, debido a las diversas oportunidades que genera y los beneficio que trae consigo. Para Gálvez, Riascos y Contreras (2014) las TIC's son un catalizador de los procesos de la empresa, por lo que se convierten en herramientas de apoyo a la administración organizacional.

Castillo, González y Vargas (2014) sostienen que la presencia de las TIC's en las empresas se destina al soporte de la gestión y a la economía, como precursor de la competitividad y la diferenciación. También citan a Coll, Mauri, y Onrubia (2008), quienes indican que las TIC representan un mecanismo de comunicación innovador, dado que se utilizan sistemas para el lenguaje visual, oral y escrito para la transmisión de mensajes a los consumidores o al personal de la empresa y proveedores. 
Por lo que las TIC representan un conjunto de tecnologías digitales, electrónicas y mecánicas que facilitan la obtención, análisis, tratamiento, comunicación y registro de la información de manera eficaz.

Según Caro et al. (2015), actualmente el uso de las TIC por parte de las micro, pequeñas y medianas empresas es más frecuente debido a la accesibilidad a su implementación. Su uso en este sector permite la supervivencia y al futuro posicionamiento de las marcas de las MiPYME, como consecuencia de que estas tecnologías facilitan el acceso a la información y a la generación de estrategias.

\subsection{LAS TIC EN LAS PYME ECUATORIANAS}

Según datos del Instituto Nacional de Estadística y Censos (2015) el 66,7\% de las empresas en el país han invertido en TIC, de dicho porcentaje el 24,6\% corresponde a empresas dedicadas a la manufactura, seguido por el 23,4\% correspondiente a empresas comerciales. El sector con mayor inversión en TIC es el sector comercial, siendo el 73,7\% de las empresas nacionales dedicadas a esta actividad las cuales han invertido en TIC.

Para el 2015, el 99\% de las empresas comerciales en Ecuador tienen al menos una computadora o netbook, el 25,5\% tienen smartphones, el 22,9\% tiene tablets y el 8\% hace uso de otros dispositivos. El 98\% de las empresas comerciales tiene acceso a internet y el 42,6\% de los trabajadores en las empresas hace uso del internet para el desarrollo de sus funciones laborales.

El 15,8\% de las empresas del sector comercio en Ecuador realizaron compras por internet y el 10,4\% realizó ventas por este medio para el año 2015. El 97,9\% de las empresas comerciales hacen uso de teléfonos celulares o servicios de comunicación, el 96,9\% se comunica a través de correo electrónico, el $60,4 \%$ de las empresas hacen uso de redes sociales para comunicarse y el 56,4\% hace uso de otros medios de comunicación. 


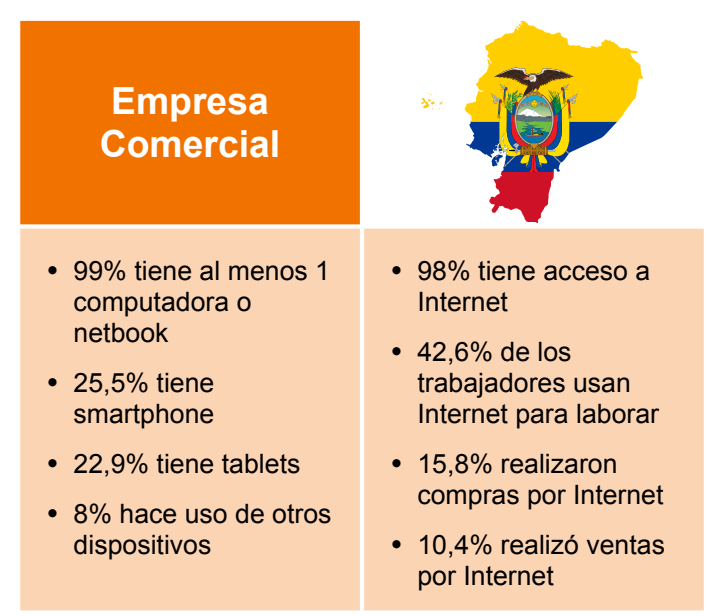

Figura 2. Datos tecnológicos de Empresas Comerciales Ecuatorianas. Fuente: elaboración propia.

En la Figura 2 se detallan datos del 2015, el 97,9\% de las empresas comerciales utilizan navegadores de internet, el 56,1\% utiliza programas de ofimática (Word, Excel, Power Point, etc.) el 39,8\% usa sistemas operativos y el 32,3\% usa otros programas de código abierto.

Según Pincay (2018), en la actualidad el uso de las TIC aplicadas a la planeación estratégica en las PYME ha crecido de modo que sus principales resultados arrojan que el 25\% de las PYME usan la computadora para la administración del negocio y solo el 3\% lo hace a través de dispositivos móviles. El 13\% de las PYME usa un ordenador para funciones de logística y el 8\% lo realiza por medio de smartphones y tablets. El 10\% usa la computadora para el monitoreo e investigación y el 2\% usa dispositivos móviles. Para la comercialización de productos el 12\% de las PYME usa la computadora y el 7\% usa los dispositivos móviles. El 59\% de las PYME usa internet para comunicación vía correo e investigación, y el 14\% lo usa para recibir facturas electrónicas.

Para Del Pozo (2015), al solo existir tres compañías de telecomunicaciones en Ecuador, la inversión que estas realicen en la adquisición de nuevas tecnologías y en el mejoramiento de sus servicios incidirá en 
la aplicación de las TIC en las empresas nacionales, debido a que será más accesibles a través de nuevos servicios o el aumento en los beneficios de los servicios actuales.

Zambrano, Párraga y Rivadeneira (2017) citan a Ca'Zorzi (2011), quien indica que las PYME apuestan a la adopción de las TIC por el ahorro en costos y la generación de beneficios, la presión ejercida por la competencia, clientes y proveedores, por decisión de la administración y el fácil uso de estas. Además, explican que la inversión en TIC no siempre impactará al crecimiento económico de la empresa, en ciertos casos, ayuda a generar valor para el cliente, valor de marca y ventaja competitiva.

Sin embargo, no todas las PYME pueden llegar a adquirir sistemas de TIC sofisticados, dado que sus necesidades son diferentes a las grandes empresas las cuales requieren una inversión más grande debido al flujo de información que manejan.

Según Párraga-Zambrano, Zambrano-Alcivar y Reyes-Trejo (2018) la aplicación de TIC en las PYME ecuatorianas es inadecuada y escasa, señalando que la mayoría de éstas no sobrepasan el nivel uno de implementación de TIC, el cual consta de las tecnologías básicas como internet, correo electrónico y teléfonos. Por tanto, no cuentan con las NTIC como sistemas ERP Enterprise Resource Planning (Planificación de Recursos Empresariales), banca electrónica, comercio electrónico y demás.

\subsection{LAS NTIC}

Las Nuevas Tecnologías de la Información y Comunicación (NTIC) se consideran a dichas tecnologías centradas en los programas informáticos o softwares, dispositivos digitales (smartphones, tablets, iwatch, entre otros) y técnicas de comunicación (redes sociales y correo) las cuales facilitan aún más los procesos organizacionales y que por otro lado son fáciles de implementar y usar. Para Sánchez (2014) las NTIC permiten a los usuarios hacer uso de la información para su análisis y el desarrollo de estrategias a distancia a través de dispositivos portátiles, consultar cuadros de mando, revisar la contabilidad, realizar operaciones bancarias y bursátiles desde cualquier punto en el que el usuario tenga acceso a internet. 
Bonete (2015) afirma que la unión entre la tecnología y la cultura de una ciudad es un evento que debe desarrollarse, de modo que en la actualidad las ciudades de primer mundo han optado por integrar las NTIC como parte de su cultura y también para facilitar a sus habitantes y turistas una mejor calidad de vida. Algunos de los ejemplos son el uso de aplicaciones para solicitar movilización, información sobre locales comerciales o de servicios, también con las aplicaciones de interacción turística, traducción en el lenguaje y procesos legales y municipales.

Según Slusarczyk (2015) la aplicación de las NTIC en las PYME del Ecuador se lleva muy poco o simplemente no se encuentran alineadas a los objetivos organizacionales. La mayor parte de estas nuevas tecnologías son implementadas y utilizadas por las grandes empresas, las cuales destinan un presupuesto a su investigación, adquisición, capacitación y uso. Las pocas PYME que tienen NTIC las han implementado por obra del azar o simplemente por recomendación de algún experto en informática, sin embargo, no se encuentran alineadas a los objetivos de la empresa y no se usan de manera óptima.

Para Flores y Ducasse (2014) la aplicación de las NTIC no se debe solo al factor económico, sino que también se debe a la falta de experticia y conocimiento sobre las tendencias tecnológicas del momento. Lo cual crea rechazo o ignorancia a la aplicación de las nuevas tecnologías, sumando a esto la preocupación de si el personal dominará dichas tecnologías y si su aplicación es tan eficaz y eficiente como prometen sus ventajas y beneficios.

\subsection{RELACIÓN DE LAS TICS Y LAS NTICS CON LA COMPETITIVIDAD}

De acuerdo a Roldán (2018), la competitividad es considerada como la "capacidad de una persona u organización para desarrollar ventajas competitivas con respecto a sus competidores y obtener así, una posición destacada en su entorno". En el caso de una empresa, es entonces una habilidad para diferenciarse de las demás de manera positiva, aquel recurso que actualmente proporciona esta ventaja es la tecnología que influye notoriamente en la decisión de compra del mercado. 
Las herramientas de las TICs y las NTIGs crean, según Mogollón (2019), en las pymes, una implementación no solamente lógica sino necesaria dada su participación en el proceso de estrategias a aplicar y así alcanzar avances frente a la competencia. Los adelantos tecnológicos son considerados clave dentro de cada organización y es de ese modo que invertir o no en TICs, influye en el éxito o fracaso de actividades comerciales.

En todo caso, en la Tabla 3 se puede apreciar que el aumento del uso de herramientas tecnológicas en Guayaquil, ha tenido un incremento considerable. De acuerdo a Observatorio de Comercio Electrónico de la Universidad Espíritu Santo (2018), las pyme reconocen que uno de los factores para medir competitividad es la implementación de nuevas tecnologías que prontamente redundarán en beneficios no solamente económicos sino también en incremento de la calidad y prestigio.

Tabla 3. Transacciones en línea en millones USD.

\begin{tabular}{|c|c|c|c|}
\hline Transacciones en línea en millones USD & $\mathbf{2 0 1 6}$ & $\mathbf{2 0 1 8}$ & Variación 2016 - 2018 \\
\hline Transacciones digitales & 136 & 184 & $35, \mathbf{2 9} \%$ \\
\hline Ventas digitales & 9428 & 11970 & $26,96 \%$ \\
\hline Transacciones ecommerce & 6 & 16 & $166,67 \%$ \\
\hline Ventas ecommerce & 646 & 1286 & $99,07 \%$ \\
\hline Total & $\mathbf{1 0 2 1 6}$ & $\mathbf{1 3 4 5 6}$ & $\mathbf{3 1 , 7 1 \%}$ \\
\hline
\end{tabular}

Fuente: (Observatorio de Comercio Electrónico de la Universidad Espíritu Santo, 2018).

Se concluye que las MiPyME son consideradas un potencial como fuente de ingresos para la población ecuatoriana, lo describen Celi y Delgado (2017). Aun teniendo debilidades como la falta de aplicación de las TICs, es imperativo mejorar la competitividad para poder sobrevivir y por ende se reconoce que esta herramienta aporta con infinidad de posibilidades para mejorar frente a la competencia y así desarrollar los objetivos planteados

Las MiPyME deben adaptarse al cambio por medio de las NTICs para mejorar su competitividad, a través de sistemas que le permitan manejar la información dentro y fuera de la organización. 


\section{CONCLUSIONES}

Es innegable que el uso de las TIC en el mundo es más común y necesario para el desarrollo de sus actividades, más aún en el ámbito empresarial, es por esto que en el caso de Ecuador y específicamente en micro y pequeñas empresas se ha abierto una puerta hace algún tiempo que impulsa a invertir e implementar en tecnologías. El aporte que realizan a las MiPYME es valioso, desde proporcionarle información oportuna que permita la generación de estrategias hasta el ascenso de posicionamiento en el mercado.

Aunque se cuenta con cifras ascendentes del uso de las TIC por parte de las micro y pequeñas empresas comerciales de Ecuador, aún es considerada insuficiente dadas que las herramientas básicas como teléfonos, correo electrónico e internet no son en su totalidad debidamente utilizados. En otras palabras y tomando un ejemplo muy cotidiano, si la empresa cuenta con línea de internet, podría utilizar banca electrónica como para transferencias de pagos a proveedores o empleados, sin embargo, este trámite sigue siendo personalizado.

El comercio electrónico como business to business, business to consumer, business to government, no están desarrollados del todo, y algunas de estas causas puede ser la desconfianza en las plataformas virtuales de las entidades financieras lo que genera que cierto segmento pierda el interés por ende desconoce sus ventajas. Esta falta de experiencia con el uso de las NTIC y en unos casos lo económico, hace que las MiPYME tomen en segundo plano su aplicación.

En los últimos 10 años el aumento del uso de las Tecnologías de la Información y Comunicación ha sido creciente tanto para las empresas como para los clientes. Paralelamente, existe un incremento de los usuarios de internet vía celular y de los planes de internet en los hogares, esto se ha vuelto un requisito para los negocios, el contar con la herramienta de las NTICs que le permita llegar y hacer conocer a sus consumidores sobre sus productos y servicios. 
El sector comercial es, por tanto, el primero en solicitar e implementar NTICs en sus micro y pequeñas empresas, dando lugar al aumento de proveedores de este servicio generando así un excelente impulso a la economía del país. Consecuentemente, las NTICs son una oportunidad para que las MiPYME promuevan sus productos y obtengan ventaja competitiva que redunde en beneficios para todo el mercado y las empresas que aún no lo han hecho, deberán adaptarse para poder sobrevivir.

\section{REFERENCIAS BIBLIOGRÁFICAS}

Bonete, F. (2015). Smart Cities y patrimonio cultural. Una integración necesaria para el desarrollo. Telos: Cuadernos de comunicación e innovación, (102), 59-66. https://dialnet.unirioja.es/servlet/articulo? codigo $=5253018$

Ca'Zorzi, A. (2011). Las TIC en el desarrollo de la PyME: Algunas experiencias de América Latina. Centro Internacional De Investigaciones Para El Desarrollo En Colaboración Con Fondo Multilateral De Inversiones/Banco Interamericano De Desarrollo. https://pymespracticas.typepad.com/files/ tic-y-pymes-en-al-final-2011.pdf

Cámara de Comercio de Quito. (2017). BOLETÍN JURÍDICO. Clasificación de las PYMES, Pequeñas y Medianas empresas. http://www.ccq.ec/wp-content/uploads/2017/06/Consulta_Societaria_Junio_2017.pdf

Caro, M. J., Leyva, C. E., Magaña, M., y Alvarado, S. (2015). Las Tic y el Ingreso en las Pymes Exportadoras del Estado de Yucatán. Atlantic Review of Economics: Revista Atlántica de Economía, 1(2), 1-29. https://dialnet.unirioja.es/servlet/articulo? codigo $=5318447$

Castillo, B., González, J. J., y Vargas, R. A. (2014). Conocimiento y uso de las TIC en las PYMES del departamento de Córdoba, Colombia. Revista Civilizar De Empresa Y Economía, 5(10), 35-53. https://doi.org/10.22518/2462909X.508 
Celi, J. E., y Delgado, G. A. (2017). Analísis De Uso de Tecnologías De Información y Comunicación, TIC en el apyo de la productividad en las Pymes de la Parroquia Tarqui del Cantón Guayaquil. Universidad de Guayaquil Facultad de Ciencias Administrativas. http://repositorio.ug.edu.ec/handle/redug/24635

Coll, G., Mauri, M. T., y Onrubia, J. (2008). Análisis de los usos reales de las TIC en contextos educativos formales: una aproximación socio-cultural. Revista Electrónica de Investigación Educativa, 10(1). https://redie.uabc.mx/redie/article/view/177/848

Del Pozo, V. (2015). Empresas y la tecnología de información y conocimiento caso Ecuador. Revista Observatorio de la Economía Latinoamericana, (210). https:/ /EconPapers.repec.org/RePEc:erv:obser$\mathrm{v}: \mathrm{y}: 2015: \mathrm{i}: 210: 28$

Delgado, D. D., y Chávez, G. P. (2018). Las Pymes en el Ecuador y sus fuentes de financiamiento. Revista Observatorio de la Economía Latinoamericana. https://www.eumed.net/rev/oel/2018/04/pymes-ecuador-financiamiento.html

Flores, G., y Ducasse, M. (2014). Entre las NTIC's y las viejas tecnologías. En Congreso Iberoamericano de Ciencia, Tecnología, Innovación y Educación, Artículo 1320, 1-14.

Gálvez, E. J., Riascos, S. G., y Contreras, F. (2014). Influencia de las tecnologías de la información y comunicación en el rendimiento de las micro, pequeñas y medianas empresas colombianas. Estudios Gerenciales, 30(133), 355-364. https://doi.org/10.1016/j.estger.2014.06.006

Guzmán, G., Guzmán, M., y Fuentes, R. (2016). Análisis del uso de las TIC en las Pymes de Guayaquil en el año 2015. OIKOS, 20(41), 109-119. http://ediciones.ucsh.cl/ojs/index.php/Oikos/ article/view/955

Hernández, R., Fernández, G., y Baptista, P. (2003). Metodología de la Investigación. McGraw Hill. https://www.esup.edu.pe/descargas/dep_investigacion/Metodologia\%20de\%20la\%20investigaci $\%$ C3 $\%$ B3n $\% 205$ ta $\% 20$ Edici $\%$ C3 $\%$ B3n.pdf 
Instituto Nacional de Estadística y Gensos. (2015). Empresas y TIC. Módulo de TIC de las Encuestas de Manufacturay Minería, Comercio Interno y Servicios. https:/ / www.ecuadorencifras.gob.ec/documentos/ web-inec/Estadisticas_Economicas/Tecnologia_Inform_Comun_Empresas-tics/2015/2015_ TICEMPRESAS_PRESENTACION.pdf

Instituto Nacional de Estadística y Gensos. (2018). Directorio de Empresas y Establecimientos 2017. https://www.ecuadorencifras.gob.ec/documentos/web-inec/Estadisticas_Economicas/DirectorioEmpresas/Directorio_Empresas_2017/Documentos_DIEE_2017/Documentos_ DIEE_2017/Principales_Resultados_DIEE_2017.pdf

Lara, E. M. (2011). Fundamentos de Investigación: Un enfoque por competencias. Alfaomega Grupo Editor.

Lopera,J., y Ramírez, C. (2010). El método analítico como método natural. Nómadas. Critical fournal of Social and furidical Sciences, 25(1), 1-28. https:/ / www.redalyc.org/articulo.oa?id=181/181 12179017

Mogollón, M. (2019, 6 de diciembre). ICTs as a decisive factor in the competitiveness of the commercial sector in the city of Guayaquil. En Proceedings of the 2nd International Conference on Information Systems \& Management Science (ISMS) 2019, Tripura University, Agartala, Tripura, India. https://papers. ssrn.com/sol3/papers.cfm?abstract_id=3512409

Muñoz, G. (2011). Cómo elaborar y asesorar una investigación de tesis (2 ${ }^{\mathrm{a}}$ ed.). Pearson Education. http:// www.indesgua.org.gt/wp-content/uploads/2016/08/Carlos-Mu\%C3\%B1oz-Razo-Como-elaborar-y-asesorar-una-investigacion-de-tesis-2Edicion.pdf

Observatorio de Comercio Electrónico de la Universidad Espíritu Santo. (2018). Comportamiento De Las Transacciones No Presenciales En Ecuador 2018: II Medición. Cámara Ecuatoriana de Comercio Electrónico. https://www.uees.edu.ec/pdf/ecommerce-2018.pdf 
Párraga-Zambrano, L. A., Zambrano-Alcivar, K. G., y Reyes-Trejo, M. A. (2018). Implementación de las tecnologías de la información y comunicación en las PYMES de la zona 4 del Ecuador y su impacto en la competitividad empresarial. Dominio de las Ciencias, 4(2), 224-238. http:/ / dx. doi. org/10.23857/dc.v4i2.808

Pincay, J. I. (2018). Modelo de planeación estratégica de tecnologías de la información en pequeñas y medianas empresas ecuatorianas. Informática y Sistemas. Revista de Tecnologías de la Informática y Telecomunicaciones, 2(1), 31-42. https://doi.org/10.33936/isrtic.v2i1.1130

Roldán, P. N. (2018). Competitividad. Economipedia. https://economipedia.com/definiciones/competitividad.html

Sánchez, J. V. (2014). Diseño de un sistema con base en las nuevas tecnologías de la información y la comunicación para la medición de la calidad y productividad de la docencia universitaria. Aibi revista de investigación, administración e ingeniería, 2(2), 2-12. https://doi.org/10.15649/2346030X.522

Slusarczyk, M. (2015). Diagnóstico de aplicación de las NTIC en las pymes de Riobamba - Ecuador. 3C TIC. Cuadernos de desarrollo aplicados a las TIC, 4(2), 145-168. https://www.3ciencias.com/ wp-content/uploads/2015/06/DIAGN\%C3\%93STICO-DE-APLICACI\%C3\%93N-DE-LASNTIC-EN-LAS-PYMES-DE-RIOBAMBA-\%E2\%80\%93-ECUADOR.pdf

Zambrano, K. G., Párraga, L. A., y Rivadeneira, F. R. (2017). Las tecnologías de la información y comunicación en las Pequeñas y medianas empresas de la Zona Norte de Manabi, Ecuador. CIDE. 